\section{MAGNETIZATION PROCESS OF HIGH ANISOTROPY COPT NANOSIZED DOTS}

N. Kikuchi', R. Murillo', J. C. Eodder', K. Mitsuzuka ${ }^{2}$ and T. Shimatsu ${ }^{2}$ (1) SMI, MESA + University of Twente, P. O. Box217 7500AE Enschede, The Netherlands

(2) RJEC, Tohoku University, Katahira 2-1-1, 980-8577 Sendai, Japan

\section{Introduction}

Magnetization reversal process of nanostructures has been studied from both practical and fundamental point of view. However, it is required to fabricate arrays on large areas for quantitative measurements due to the lack of sensitivity of conventional magnetometory We have proposed anomalous Hall effect (AHE) measurement as an altemative method which realize both of simplicity of fabrication and high sensitivity of measurement $[1,2]$ In this paper, we report on the results of measuring the nanodot array of $\mathrm{CO}_{80} \mathrm{Pt}_{20}$ with large perpendicular anisotropy.

\section{Experimental Procedure}

$\mathrm{Co}_{80} \mathrm{Pt}_{20}$ films are deposited on a thermally oxidized $\mathrm{Si}$ substrate by magnetron sputtering [3] The film structure is $\mathrm{Pt}(2 \mathrm{~nm}) / \mathrm{Co}_{80} \mathrm{Pt}_{20}(20 \mathrm{~nm}) / \mathrm{Ru}(20 \mathrm{~nm}) / \mathrm{Pt}(10 \mathrm{~nm}) / \mathrm{Ta}(5 \mathrm{~nm}) / \mathrm{SiO}_{2} / \mathrm{Si}$. The hep-CoPt (002) plane is epitaxially grown on the Ru buffer layer, and the easy axis of the film is normal to the film plane. The uniaxial anisotropy constant $K_{u}$ and saturation magnetization $M_{s}$ are $1.27 \times 10^{7}(\mathrm{erg} / \mathrm{cc})$ and $1200(\mathrm{emu} / \mathrm{cc})$, respectively. A dot array is fabricated by using laser interference lithography (LIL) and ion beam etching with $\mathrm{Ar}^{+}$. The etching process is stopped in the middle of the Ru layer in order to use nonmagnetic buffer layers as electrode for the AHE measurement. The dot diameter and the periodicity are 120 $\mathrm{nm}$ and $300 \mathrm{~nm}$, respectively. With these dimensions, interaction between dots can be negligible. Magnetic properties of the array are examined by AHE measurement.

Results

Figure 1 shows AHE curves of continuous film and a dot array. Significant increase of coercivity is observed after patterning. From minor loop measurement it is confirmed that all the magnetization change in perpendicular field is irreversible. The result indicates that the slope of hysteresis curve represents the distribution of switching field of each dot. In Fig. 2, remanent coercivity $\left(H_{\mathrm{c}}{ }^{\mathrm{L}}\right)$ of the array is plotted as function of angle between applied field and film nomal. $H_{\mathrm{c}}^{\mathrm{r}}$ shows minimum value at $\theta=45^{\circ}$, which is about $70 \%$ of ${H_{\mathrm{c}}}_{\mathrm{c}}$ at $\theta$ $=0^{\circ}$. This behavior suggests that magnetization rotation process of a reversed nucleus determines switching field of the dot, while domain wall motion is dominant in $\mathrm{Co} / \mathrm{Pt}$ multilayer dots [2], as shown as dashed line in Fig. 2. In Fig. 3, coercivity of the array is plotted as function of sweep rate of external field. The sweep rate is varied from 0.1 to 50 (Oe/s). Coercivity slightly increases as increase of sweep rate. Energy barrier height of magnetization reversal is evaluated by assuming Neel-Arrhenius model. The fitting result is indicated as a dotted line in Fig. 3. The barrier height $E_{\mathrm{b}}$ is estimated as $7 \times 10^{-11}$ (erg), which corresponds to the energy more than $10^{3} k_{\mathrm{B}} T$ at toom temperature. This value is one order larger than the barrier height obtained in $\mathrm{Co} / \mathrm{Pt}$ multilayer dots [2]. By assuming a relation of $E_{\mathrm{b}}=K_{\mathrm{u}} \times V_{\mathrm{sw}}$, switching volume $V_{\mathrm{sw}}$ is estimated as $(17 \mathrm{~nm})^{3}$, which is about $1 \%$ of the volume of the whole dot.

In summary, we have investigated the magnetization reversal process of the CoPt dot and found that the magnetization reversal of the dot is initiated by the rotation process of a nucleus with the volume of $(17 \mathrm{~nm})^{3}$.

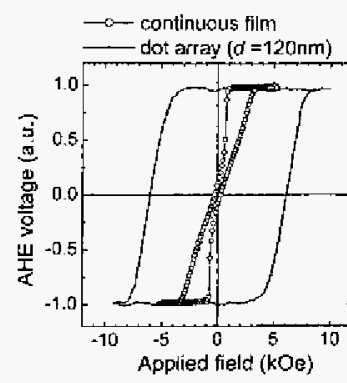

Fig. 1 AHE hysteressis curve of CoP1 continuous film and dot array of $120 \mathrm{~nm}$ in diameter.

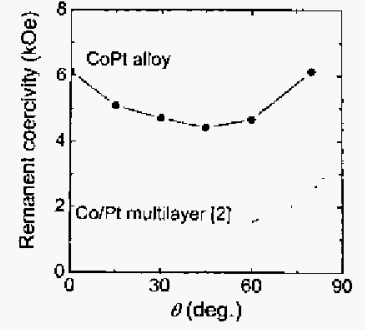

Fig. 2 Remanent coercivity of Copt dot array as function of angle berween external tield and the film normat.

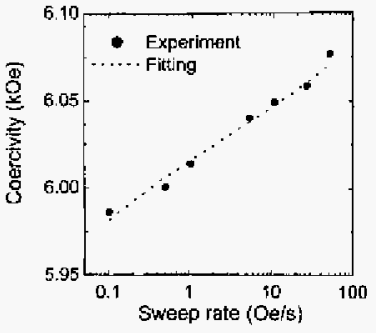

Fig. 3 Coercivity of CoPt dot array as function of sweep rate of external field.
Reference

[1] N. Kikuchi, S. Okamoto, O. Kitakami, Y. Shimada, and K. Fukamichi, Appl. Phys. Lett. 82, 4313 (2003) [2] N. Kikuchi, R. Murilio, and J. C. Lodder, (submitted, proceedings the $49^{\text {th }}$ MMM conference) [3] T. Shimatsu, H. Sato, T. Oikawa, Y. Inaba, O. Kitakami, S. Okamoto, H. Aoi, H. Muraoka, Y. Nakamura, IEEE Trans Magil., 40, $2483(2004)$ 